\title{
Embryology and Uterine Abnormalities, Shapes and Miscarriage Risks
}

\author{
Mohamed Zarqaoui ${ }^{1}$, Mustafa Zakaria ${ }^{2 *}$ and Noureddine Louanjli ${ }^{3}$ \\ ${ }^{1}$ General Medical Coordinator of IRIFIV Fertility Center, Head of the ART IRIFIV \\ Scientific Research Group (AISRG) Casablanca, Morocco \\ ${ }^{2}$ Consultant at IRIFIV Fertility Center, Administrative Deputy and Writer for the ART \\ IRIFIV Scientific Research Group (AISRG) Casablanca, Morocco \\ ${ }^{3}$ Head of LABOMAC Laboratory of Clinical Analysis and Assisted Reproductive \\ Technology IRIFIV Fertility Center, ANFA Fertility Center - Executive Vice President of \\ the ART IRIFIV Scientific Research Group (AISRG) Casablanca, Morocco
}

*Corresponding Author: Mustafa Zakaria, Consultant at IRIFIV Fertility Center, Administrative Deputy and Writer for the ART IRIFIV Scientific Research Group (AISRG) Casablanca, Morocco.
Received: September 18, 2020

Published: September 30, 2020

(C) All rights are reserved by Mustafa

Zakaria., et al.

\section{Abstract}

An abnormal uterus can sometimes be a risk factor for miscarriage and, in some cases, a cause of recurrent miscarriages. however, only some types of uterine malformations increase the risk of miscarriage and require treatment. others may not cause any problems with pregnancy at all. about $18 \%$ of women who have recurrent miscarriages have some type of uterine abnormality. Some uterine malformations are present from birth, while others develop during adulthood. most often, women with uterine abnormalities do not have any symptoms and are not aware of these malformations before they become pregnant. Diagnosis of congenital uterine malformations usually comes after a hysterosalpingogram (HSG), but this test can miss some conditions, such as uterine septum. Any abnormal HSG should be followed by a hysteroscopy. A uterine septum (septate uterus) is the most common congenital uterine anomaly, comprising roughly $55 \%$ of Mullerian duct anomalies. Congenital means that it is present at birth. A uterine septum is a band of fibrous tissue that partially or completely divides the uterus, usually without a good blood supply. If a fertilized egg implants on the septum, the placenta is unable to grow properly and miscarriage is likely, for women who have a septate uterus, the risk of miscarriage is significant. In fact, in one study nearly $67 \%$ of the women in the study with a septate uterus experienced pregnancy loss, for women who do not miscarry, a septate uterus may increase the risk of preterm birth. In fact, the same study found that the likelihood of having a full-term, normal-sized baby was only on $25 \%$. treatment is usually minor surgery, performed during a hysteroscopy, to remove the abnormal tissue. this usually works extremely well to resolve the problem and allow women to successfully carry a full-term pregnancy.

Keywords: Embryology; Uterine Abnormalities; Miscarriage

\section{Introduction}

Embryology

The uterus is formed from two paramesonephric ducts (Mullerian ducts). The caudal two thirds of these ducts form the uterus, whereas the upper third gives rise to the fallopian tubes.
Failure or arrest of development during any of the following three stages will lead to uterine malformation (Figure 1).

At 6 - 9 weeks of gestation: Müllerian ducts appear and there is a caudal midline fusion and connection with the urogenital sinus. So, failure of development of the Müllerian ducts during this period leads to uterine aplasia (Figure 2). 


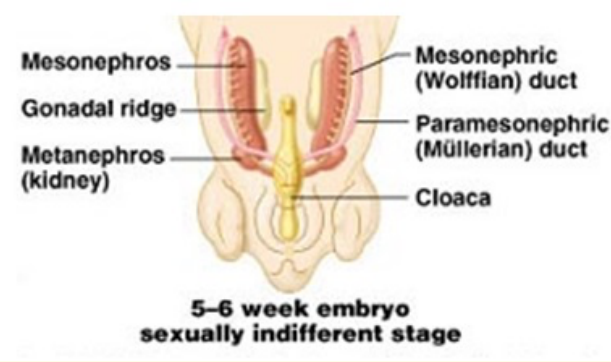

Figure 1

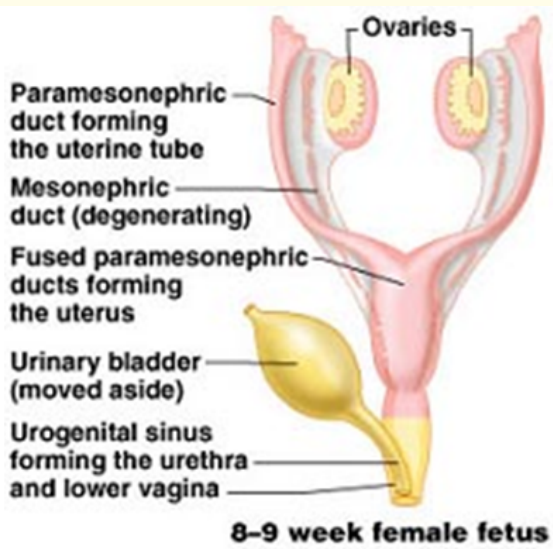

Figure 2

At 10 - 13 weeks of gestation: There is an upward fusion of the caudal parts of the Müllerian ducts; Any failure of fusion during this period of time leads to uterine duplications (uterus didelphys, bicornuate uterus).

At 14 - 18 weeks of gestation: The normal resorption of the medial septum initially separating the caudal parts of the Müllerian ducts takes place in order to form the utero-vaginal channel which is the origin of the uterine cavity and the superior $2 / 3$ of the vagina. Failure of resorption of the midline septum leads to uterine septation (septate uterus). Normally, the resorption of the septum occurs caudad to cephalad; It begins at the level of the cervix and continues up to the uterine fundus. Septate uterus is never combined with another anomaly of the genito-urinary tract.

Generally, the uterus is formed by the joining of bilateral paramesonephric ducts. Failure to join or incorrect joining results in various types of duplications of the uterus. A uterus didelphys is a completely doubled uterus and doubled vagina. A bicornuate uterus is one in which the uterus has two horns ending in a common vagina.

Causes for the failure to fuse are not known. The condition is less common than these other uterine malformations: arcuate uterus, septate uterus, and bicornuate uterus. It has been estimated to occur in $1 / 3,000$ women.

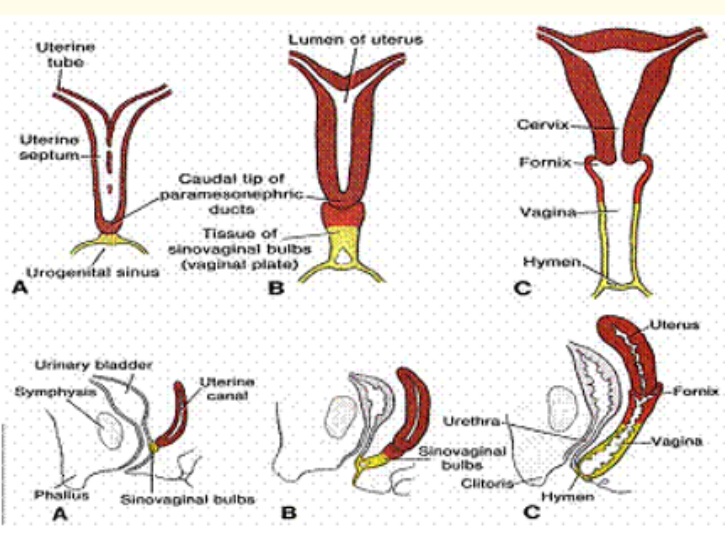

Figure 3

Female genital tract developed from 3 origins:

1. Ovaries from the germ cells that migrate from the yolk sac into the mesenchyme of the peritoneal cavity and developed into ova and supporting cells.

2. Lower third of vagina developed from the ascending Sino vaginal bulb.

3. Uterus, fallopian tubes and upper two thirds of vagina developed from the fusion of two Müllerian ducts and this is our subject for discussion.

The three stages

\section{Organogenesis}

If one of the tubes fail to develop it results in unicornuate uterus if both this cause uterine agenesis or hypoplasia.

\section{Fusion}

Two kinds of fusion:

1. Lateral fusion process by which the two Müllerian ducts fuse together to form the uterus, tubes and cervix failure of this process cause bicornuate or didelphys uterus. 
2. Vertical fusion formation of vagina completed by fusion of the lower part of Müllerian duct that form the upper two thirds and the ascending Sino vaginal bulb that forms the lower one third incomplete vertical fusion lead to imperforated hymen.

\section{Septal resorption}

Normally the median part of the fused Müllerian ducts forms septum that resorped to form single uterine cavity failure of this resorption lead to septate uterus complete or partial.

Frequency: It occurs in $0.1-0.5 \%$ in healthy fertile population.

\section{Clinical presentation}

It differs in each patient according to the type of abnormality but nearly the most common presentations are:

- Infertility

- Recurrent abortion

- Preterm labor

- Intra uterine growth retardation

- Primary amenorrhea in imperforated hymen and haematocolpus.

\section{Diagnosis}

Clinical history and physical examination cannot give much about the diagnosis so the best way is by using imaging techniques: Hysterosalpingography.

The most common tool to assess the uterine cavity and opening of the fallopian tube is still the hysterosalpingogram. Uterine cavity defects such as fibroids, polyps and synechiae can be clearly demonstrated with HSG. Contrast media used are oil-based or waterbased. It has been claimed that pregnancy rate after using oil-based media is higher. It is clear today that water-soluble media reduces inflammatory reactions, especially granulomatous inflammation, and the risk of oil embolism.

Have false positive results of $38 \%$ and falls negative results of $28 \%$ it also cannot assess the outer fundal contour making it impossible to differentiate between septate and bicornuate uterus.

Special criteria putted in mind to confirm the diagnosis by HSG

Intercornual distance: The distance between the distal ends of the horns.

- $\quad$ Septate uterus: If less than $2 \mathrm{~cm}$ means
- $\quad$ Bicornuate uterus: If more than $4 \mathrm{~cm}$ means

- $\quad$ Normal uterus: 2 - $4 \mathrm{~cm}$ means

- Intercornual angle: Formed by the most medial aspects of the two uterine hemi cavities

- $\quad$ Septate uterus: If less than 60 means

- Bicornuate uterus: If more than 60 means.

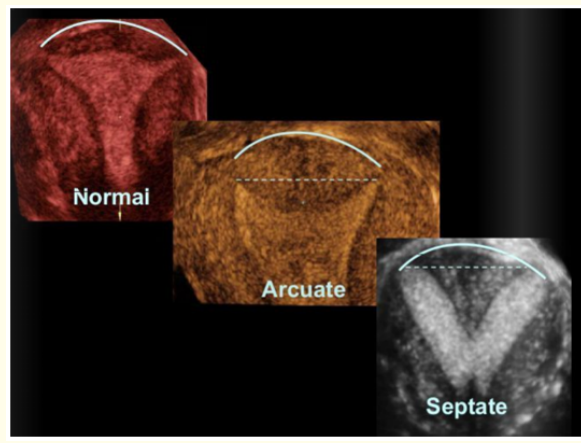

Figure 4

T shaped uterus means DES exposure.

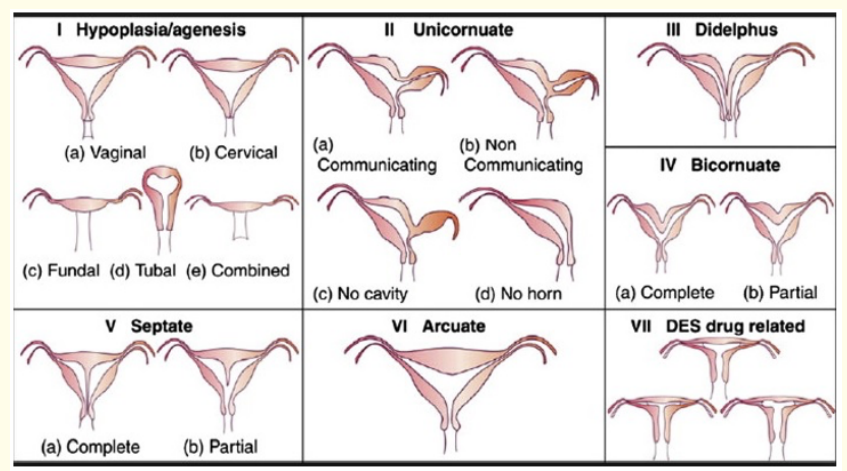

Figure 5

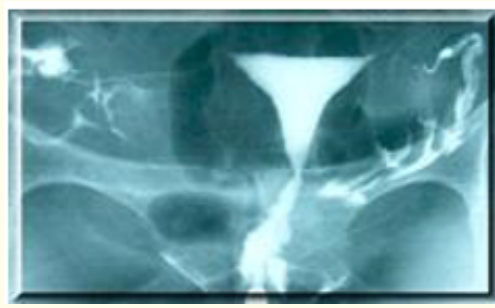

Figure 6: Normal examination. 

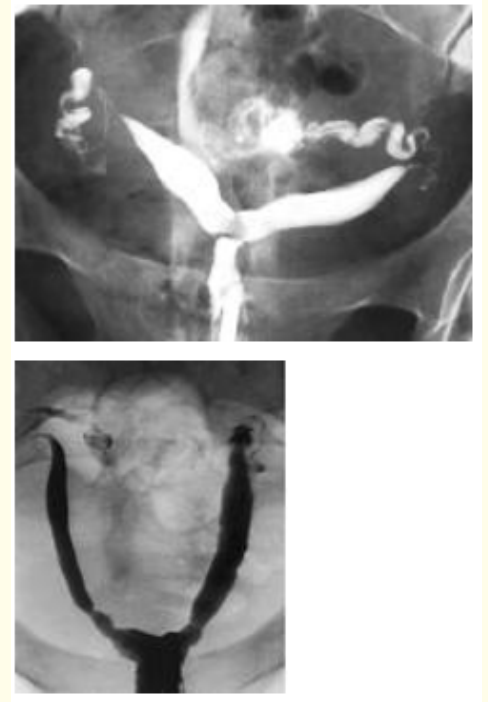

Figure 7: Bicornuate uterus.

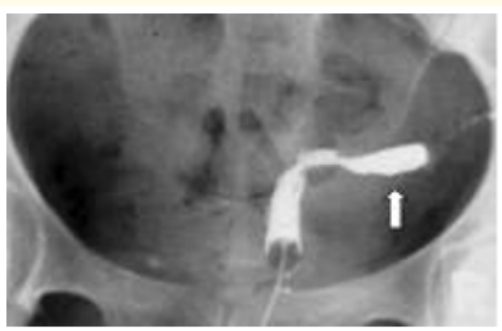

Figure 8: Unicornuate uterus.

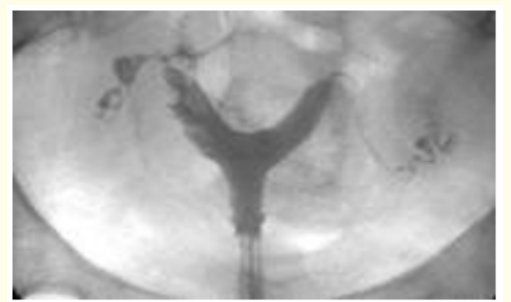

Figure 9: Septate uterus.

\begin{tabular}{|l|c|}
\hline \begin{tabular}{|} 
Prevalence of uterine anomalies among \\
consecutive patients with RM.
\end{tabular} \\
\hline \begin{tabular}{|cc|} 
\%occurrence $(\mathrm{n})$ \\
\hline Total frequency of anomalies
\end{tabular} \\
\hline Congenital anomalies & $19.5(176)$ \\
\hline Bicornuate uterus & $6.7(61)$ \\
\hline Didelphic uterus & $0.8(7)$ \\
\hline Septate uterus & $0.2(2)$ \\
\hline T-shaped uterus & $4.8(43)$ \\
\hline Unicornuate uterus & $0.3(3)$ \\
\hline Acquired anomalies & $0.7(6)$ \\
\hline Adhesions & $13.3(120)$ \\
\hline Fibroid(s) & $4.1(37)$ \\
\hline Polyp(s) & $6.4(58)$ \\
\hline & $3.2(29)$ \\
\hline
\end{tabular}

Figure 10
MRI

MRI (magnetic resonance imaging).

A procedure in which radio waves and a powerful magnet linked to a computer are used to create detailed pictures of areas inside the body.

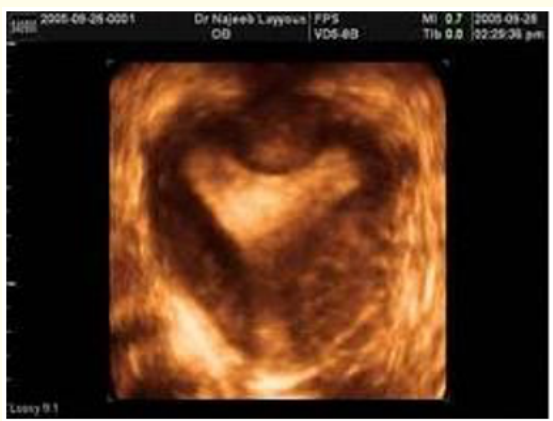

Figure 11: Arcuate uterus.
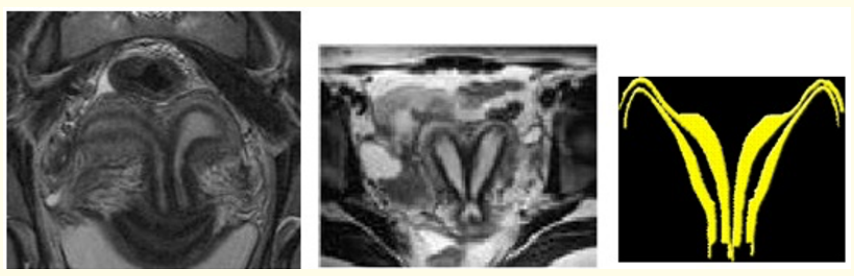

Figure 12: Uterus didelphys.
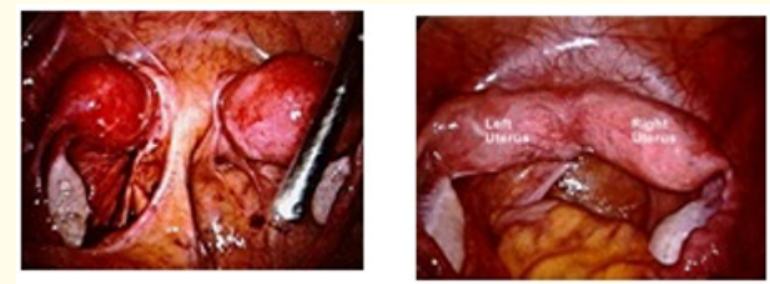

Figure 13: Hysteroscopy.

Hysteroscopy is a simple procedure to visualize the cervical canal and the inside of the uterus using a thin, lighted, flexible tube designated a hysteroscope. The device is inserted through the vagina. It can be used as a diagnostic, as well as a therapeutic tool. The procedure can be performed with or without a local anesthetic, especially if it is used for a diagnostic procedure only. Occasionally this is performed under general anesthesia, usually to remove a polyp, fibroid, adhesions or for biopsy.

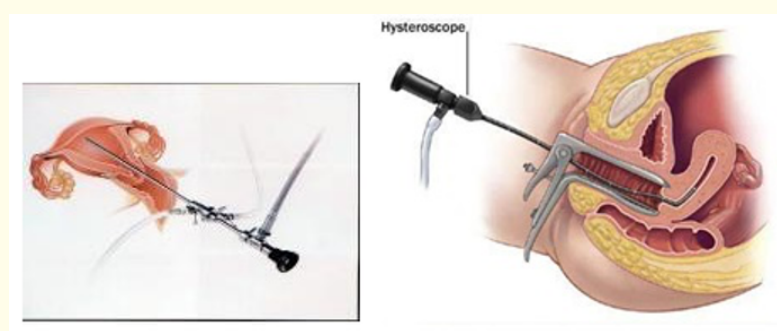

Figure 14: The hysteroscope inserted into the uterus through the cervix. 


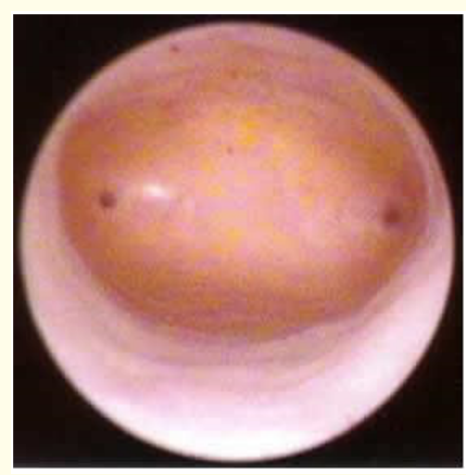

Figure 15: Normal hysteroscopic view for comparison.

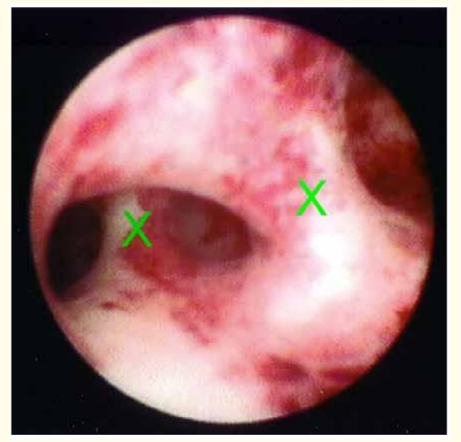

Figure 16: Uterine septum at hysteroscopy.

\section{Three-dimensional ultrasound}

It provide information that is the same or better than that tained with MRI less expensive and readily available in most centers which enables expedited diagnosis.

When combined with saline infusion 3 D ultrasound yield information similar to that obtained by HSG.

When scanning a gynecology patient especially if a uterine abnormality is imaged the kidneys need to be assessed.

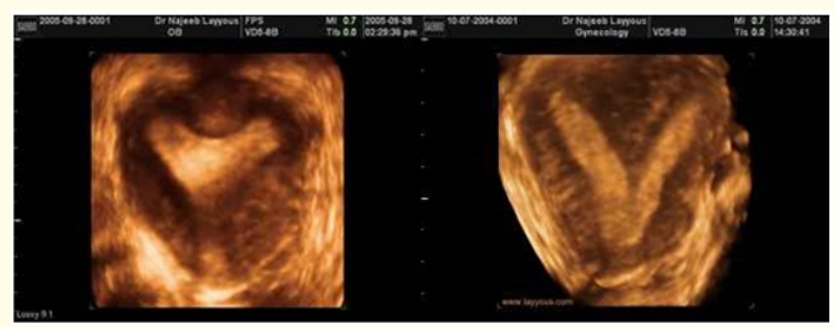

Figure 17
Classification and surgical treatment

\section{Anatomy}

Müllerian duct anomalies are categorized most commonly lasses according to the American Fertility Society (AFS) Classification Scheme (1988 into 7) as follows.

\section{Class I (hypoplasia/agenesis)}

This class includes entities such as uterine/cervical agenesis or hypoplasia. The most common form is the Mayer-RokitanskyKuster-Hauser syndrome, which is combined agenesis of the uterus, cervix, and upper portion of the vagina. Patients have no reproductive potential aside from medical intervention in the form of in vitro fertilization of harvested ova and implantation in a host uterus occur in 15-40\%and also some times skeletal abnormalities.

Diagnosis of vaginal agenesis

- $\quad$ Presented as primary amenorrhea

- Normal secondary sex characteristics

- Pelvic examination reveals patulous urethra

- Vagina can be completely absent or shunt vaginal pouch can be present

- Uterus cannot be palpated on rectal examination

- Ultra sound show absent uterus and sometimes renal abnormalities.

Laparoscopy is not indicated unless the diagnosis cannot be determined by the above findings.

\section{Treatment}

1. Non surgical treatment by using graduated dilators may take several months to few years before a functional vagina is formed so surgery remains the most effective method of treatment.

2. Surgical treatment by vaginoplasty the aim is to create new vagina surgical treatment should be considered only when the patient wishes to become sexually active and is highly motivated to use vaginal prosthesis for several months after surgery there are various methods of vaginal reconstruction we will discuss some of these methods:

- $\quad$ Full thickness skin graft

- Transposition flaps.

\section{Class II (unicornuate uterus)}

A unicornuate uterus is the result of complete, or almost complete, arrest of development of 1 Müllerian duct If the arrest is in- 
complete, as in $90 \%$ of patients, a rudimentary horn with or without functioning endometrium is present. If the rudimentary horn is obstructed, it may come to surgical attention when presenting as an enlarging pelvic mass. If the contra lateral healthy horn is almost fully developed, a full-term pregnancy is believed to be possible (see didelphys uterus).

\section{Surgical treatment}

Women with unicornuate uterus are not generally considered for reconstruction metroplasty the only surgical indication is the presence of endometrium In the accessory horn and this can be removed by laparoscopic hemi hysterectomy while the operation not indicated for rudimentary horn lacks and endometrium.

\section{Class III (didelphys uterus)}

This anomaly results from complete nonunion of both Müllerian ducts the individual horns are fully developed and almost normal in size. Two cervices are inevitably present. A longitudinal or transverse vaginal septum may be noted as well. Didelphys uteri have the highest association with transverse vaginal septa but septa also may be observed in other anomalies. Consider metroplasty; however, since each horn is almost a fully developed uterus, patients have been known to carry pregnancies to full term treatment is different according to the presentation:

1. Uterine didelphys with obstructed unilateral vagina full excision on marsuplization of the vaginal septum. Also, laparoscopy preferred to be done intra operatively to treat associated endometriosis or adhesions if present.

2. Uterus didelphys non obstructed these patients are not candidates for surgical unification. Fortunately, they have minimum associated problems and the fertility is good.

\section{Class IV (bicornuate uterus)}

A bicornuate uterus results from partial nonunion of the Müllerian ducts the central myometrium may extend to the level of the internal cervical os (bicornuate unicollis) or external cervical os (bicornuate bicollis). The latter is distinguished from didelphys uterus because it demonstrates some degree of fusion between the two horns, while in classic didelphys uterus, the two horns and cervices are separated completely. In addition, the horns of the bicornuate uteri are not fully developed; typically, they are smaller than those of didelphys uteri. Some patients are surgical candidates for metroplasty surgical treatment. Although numbers of metroplasty procedures are available the strassmann procedure is the surgical treatment of choice for unifying the bicornuate and didelphys uteri this is simply explained by fusing the fundi of the uteri if 2 cervices are present their unification is not recommended. Hysteroscopy resection is contraindicated in this setting because it can result in uterine perforation.

\section{Class V (septate uterus)}

A septate uterus results from failure of resorption of the septum between the two uterine horns. The septum can be partial or complete, in which case it extends to the internal cervical os histologically, and the septum may be composed of myometrium or fibrous tissue. The uterine fundus is typically convex but may be flat or slightly concave ( $<1$-cm fundal cleft). Women with septate uterus have the highest incidence of reproductive complications. Differentiation between a septate and a bicornuate uterus is important because septate uteri are treated using transvaginal hysteroscopic resection of the septum, while if surgery is possible and/or indicated for the bicornuate uterus, an abdominal approach is required to perform metroplasty.

\section{Surgical treatment}

The treatment of choice is transcervical lyses of uterine septum combined with concurrent laparoscopy to reduce the risk of uterine perforation. The operation done by hysteroscopy which is introduced through the cervix and cutting of the septum done and preferably to put IUCD for at least 2 months to prevent intrauterine adhesions formation others found this is unnecessary and may provoke local inflammation with subsequent synechiae.

\section{Class VI (arcuate uterus)}

An arcuate uterus has a single uterine cavity with a convex or flat uterine fundus, the endometrial cavity, which demonstrates a small fundal cleft or impression $(>1.5 \mathrm{~cm}$ ). The outer contour of the uterus is convex or flat. This form is often considered a normal variant since it is not significantly associated with the increased risks of pregnancy loss and the other complications found in other subtypes. This abnormality doesn't need any interference because researches found there is no difference in reproductive outcome compared with normal.

\section{Class VII (diethylstilbestrol-related anomaly)}

Several million women were treated with diethylstilbestrol (DES; an estrogen analog prescribed to prevent miscarriage) from 1945 - 1971. The drug was withdrawn once its teratogenic effects 
on the reproductive tracts of male and female fetuses were understood. The uterine anomaly is seen in the female offspring of as many as $15 \%$ of women exposed to DES during pregnancy. Female fetuses who are affected have a variety of abnormal findings that include uterine hypoplasia and a T-shaped uterine cavity. Patients also may have abnormal transverse ridges, hoods, stenosis of the cervix, and adenosis of the vagina with increased risk of vaginal clear cell carcinoma. Imaging findings are path gnomonic for this anomaly) [1-6].

\section{Conclusion}

Not all the types of uterine abnormalities can affect fertility and also not all the abnormalities correctable surgically Uterine Abnormalities (Müllerian duct anomalies) are an uncommon but often treatable cause of infertility. Patients with Müllerian duct anomalies are known to have a higher incidence of infertility, repeated first trimester spontaneous abortions, fetal intrauterine growth retardation, fetal malposition, preterm labor, and retained placenta. The role of imaging is to help detect, diagnose, and distinguish surgically correctable forms of Müllerian duct anomalies from inoperable forms. In some correctable lesions, the surgical approach is altered based on imaging findings.

\section{Bibliography}

1. Kupesic S. "Clinical implications of sonographic detection of uterine anomalies for reproductive outcome". Ultrasound in Obstetrics and Gynecology 18.4 (2001): 387-400.

2. Laifer-Narin S., et al. "False-normal appearance of the endometrium on conventional transvaginal sonography: comparison with saline hysterosonography". American Journal of Roentgenology 178.1 (2002): 129-133.

3. Godinjak Z and Idrizbegovic E. "Should diagnostic hysteroscopy be a routine procedure during diagnostic laparoscopy in infertile women?" Bosnian Journal of Basic Medical Sciences 8.1 (2008): 44-47.

4. Golan A., et al. "Diagnostic hysteroscopy: its value in an in-vitro fertilization/embryo transfer unit". Human Reproduction 7 (1992a): 1433-1434.

5. Guimaraes Filho HA., et al. "Prevalence of uterine defects in habitual abortion patients attended on at a university health service in Brazil". Archives of Gynecology and Obstetrics 274.6 (2006b): 345-348.
6. Saravelos SH., et al. "Prevalence and diagnosis of congenital uterine anomalies in women with reproductive failure: a critical appraisal". Human Reproduction Update 14.5 (2008): 415429.

\section{Assets from publication with us}

- Prompt Acknowledgement after receiving the article

- Thorough Double blinded peer review

- Rapid Publication

- Issue of Publication Certificate

- High visibility of your Published work

Website: www.actascientific.com/

Submit Article: www.actascientific.com/submission.php

Email us: editor@actascientific.com

Contact us: +919182824667 